
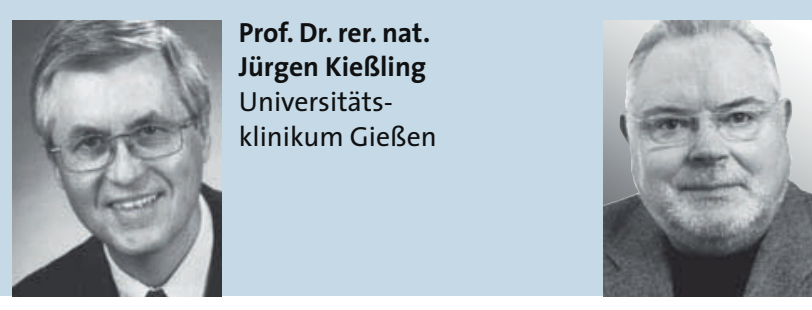

Dr. med.

Dieter Leithäuser

HNO-Arzt, Warburg

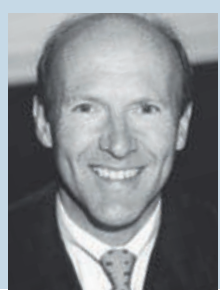

Prof. Dr. med.

Ralph Mösges

Universität Köln

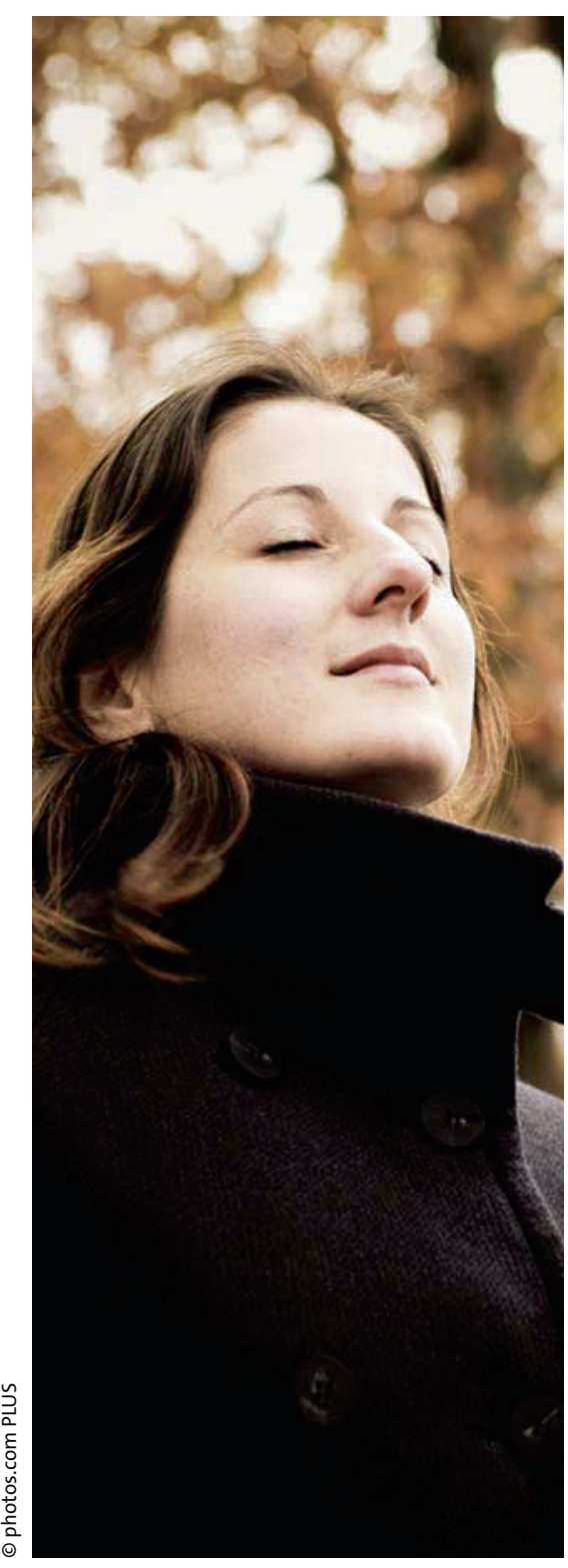

Frei durchatmen: Mit Radiofrequenzinduzierter Thermotherapie bessert sich die nasale Obstruktion bei Nasenklappeninsuffizienz.

\section{Nasenklappe stabilisieren: neue Operationsmethode im Test}

\begin{abstract}
Häufig ist für die nasale Obstruktion eine insuffiziente Nasenklappe verantwortlich. Ob sich zur operativen Behandlung der Einsatz der Radiofrequenz-induzierten Thermotherapie eignet, wurde in einer klinischen Studie untersucht.
\end{abstract}

$\mathrm{D}$ ie Nasenklappe ist definiert als Region zwischen Apertura piriformis, lateralem Anteil des Flügelknorpels, Septumknorpel und dem anterioren Teil der unteren Nasenmuschel. Hier besteht der höchste Luftwiderstand bei der nasalen Atmung. Eine der häufigsten Ursachen für nasale Obstruktion besteht in der Insuffizienz dieser Klappe: Bei Inspiration kollabiert das Weichteilgewebe, typisch ist dabei das Anlegen des Nasenflügels bei festem Einatmen durch die Nase.

In einer türkischen Studie wurde geprüft, ob die Radiofrequenz-induzierte Thermotherapie (RFITT) hier erfolgreich eingesetzt werden kann. Der Autor schloss 28 Patienten in seine Studie ein. Die Studienteilnehmer (zwölf Frauen und 16 Männer, Durchschnittsalter 29,5 Jahre) litten an nasaler Obstruktion bei inspiratorischem Kollaps der Nasenklappe. Andere Ursachen für eine Obstruktion wie Muschelhypertrophie, nasale Polypen oder Septumdeviation bestanden nicht. Der Eingriff erfolgte in Lokalanästhesie. Durch Dissektion wurde im Vestibulum nasi ein größeres Schleimhautpolster im Bereich zwischen dem lateralen Flügelknorpelanteil und der Apertura piriformis gebildet. Anschließend erfolgte hier eine Energieapplikation mittels RFITT. Ziel der Operation war die Fibrosierung und Retraktion dieser Schleimhautregion im Wundheilungsverlauf mit einer resultierenden Weitstellung der Nasenklappe.

Die Operation zeigte nach 16 Wochen einem signifikanten Rückgang der Obstruktion. Die Patienten gaben in einer visuellen Analogskala eine Verringerung der Obstruktion von 8,2 auf 3,4 Punkte (linke Nasenöffnung) beziehungsweise von 8,9 auf 4,1 Punkte (rechte Nasenöffnung) an. Schwere oder länger andauernde Komplikationen traten nicht auf - nur drei Patienten klagten während und kurz nach der Behandlung über milde bis moderate Schmerzen, bei einem Patienten zeigte sich ein umschriebenes Ödem.

Fazit: In dieser kleinen Studie besserte sich durch die Radiofrequenz-induzierte Thermotherapie die nasalen Obstruktion bei Nasenklappeninsuffizienz. Der Behandlungserfolg sollte in einer kontrollierten Studie mit höherer Teilnehmerzahl bestätigt werden.

Seren E. A new surgical method of dynamic nasal valve collapse.

Arch Otolaryngol Head Neck Surg 2009; 135: 1010-4 